\title{
Calculation of reflected and transmitted powers of a metamaterial waveguide structure using MAPLE software
}

\section{Mohammed Shabat, Muin Ubeid, Mohammed Sid-Ahmed}

Mohammed M. Shabat, Muin F. Ubeid, Mohammed O. Sid-Ahmed, "Calculation of reflected and transmitted powers of a metamaterial waveguide structure using MAPLE software," Proc. SPIE 9289, 12th Education and Training in Optics and Photonics Conference, 92891L (17 July 2014); doi: $10.1117 / 12.2070526$

Event: 12th Education and Training in Optics and Photonics Conference, 2013, Porto, Portugal 


\title{
Calculation of reflected and transmitted powers of a metamaterial waveguide structure using MAPLE software
}

\author{
Mohammed M. Shabat ${ }^{1 * a}$, Muin F. Ubeid ${ }^{\mathrm{a}}$, Mohammed O. Sid-Ahmed ${ }^{\mathrm{b}}$ \\ ${ }^{a}$ Department of Physics, Faculty of Science, Islamic University of Gaza, P.O.Box 108, Gaza, Gaza \\ Strip, Palestinian Authority, ${ }^{*}$ email: shabatm@gmail.com \\ ${ }^{b}$ Department of Physics, Faculty of Science, Sudan University of Science and Technology, The \\ Republic of The Sudan
}

\begin{abstract}
Consider a waveguide structure consisting of a pair of metamaterial and dielectric slabs inserted in vacuum. A plane polarized wave is obliquely incident on it. Metamaterials (sometimes termed left-handed materials (LHMs)) are materials whose real parts of permittivity $\varepsilon$ and permeability $\mu$ are both negative and consequently have negative index of refraction. The transmission of the electromagnetic waves through the structure is analyzed theoretically and numerically with the emphasis on the dissipation factor. Maxwell's equations are used to determine the electric and magnetic fields of the incident waves in each region. Then, Snell's law is applied and the boundary conditions of the fields are imposed at each layer interface to obtain a number of equations with unknown parameters. The MAPLE is used to solve these equations for the unknown parameters to calculate the reflection and transmission coefficients. These coefficients are used to determine the reflected, transmitted and loss powers of the structure. In the numerical results the mentioned powers are computed and illustrated as a function of frequency, angle of incidence and slab thickness when the dissipation factor changes.
\end{abstract}

Key words: Electromagnetic waves, frequency, left-handed material, dissipation factor, refractive index, transmitted power.

\section{INTRODUCTION}

Metamaterials (sometimes termed left-handed materials (LHMs)) are materials whose permittivity $\varepsilon$ and permeability $\mu$ are both negative and consequently have negative index of refraction. These materials are artificial and theoretically discussed first by Veselago [1] over 40 years ago. The first realization of such materials, consisting of split-ring resenators (SRRs) and continuous wires, was first introduced by Pendry [2,3]. Regular materials are materials whose $\varepsilon$ and $\mu$ are both positive and termed right handed materials (RHMs). R. A. Shelby et al [4] have studied negative refraction in LHMs. I. V. Shadrivov [5] has investigated nonlinear guided waves in LHMs. N. Garcia et al [6] have shown that LHMs don't make a perfect lens. Kong [7] has provided a general formulation for the electromagnetic wave interaction with stratified metamaterial structures. M. M. Shabat et al [8] have discussed Nonlinear TE surface waves in a left-handed material and magnetic super lattice waveguide structure. I. Kourakis et al [9] have investigated a nonlinear propagation of electromagnetic waves in negative-refraction index LHM. M. F. Ubeid et al [10] have demonstrated the effect of negative permittivity and permeability on the transmission of electromagnetic waves through a structure containing left-handed material. H. Cory et al [11] and C. Sabah et al [12] have estimated high reflection coatings of multilayered structure. Oraizi et al [13] have obtained a zero reflection from multilayered metamaterial structures.

In this paper we consider a structure consisting of LHM and dielectric slabs inserted in vacuum. A plane polarized wave is obliquely incident on it. Tow cases of the LHM are considered, loss-less case and loss case. Maxwell's equations are used to determine the electric and magnetic fields in each region. Then, Snell's law is applied and boundary conditions are imposed at each interface to obtain a number of equations with unknown parameters. The equations are solved for the unknown parameters by a MAPLE software to calculate the reflection and transmission coefficients. These coefficients are used to determine the reflected, transmitted and loss powers of the structure. The effect of many

*shabatm@gmail.com; phone +97082860800; fax +97082860700

12th Education and Training in Optics and Photonics Conference, edited by

Manuel F. P. C. Martins Costa, Mourad Zghal, Proc. of SPIE Vol. 9289, 92891L

(C) 2014 SPIE, OSA, IEEE, ICO · doi: 10.1117/12.2070526 
parameters like frequency, angle of incidence etc. on the mentioned powers are studied in details by changing the dissipation factor of the LHM. Throughout the computations, the frequency dependence of permittivity and permeability of the LHM are taken into account. The numerical results are in agreement with the law of conservation of energy given by $[11,14,15]$. It is also noticed that the numerical results of Figure 4 is similar to Figure 8.6 obtained by [14], this is another evidence for validity of the performed computations. The propagation of electromagnetic waves through a structure containing LHM and dielectric is considered by many previous studies. Our work is concentrated on the powers of the structure and the role of properties of the dissipation factor of LHM layer. Thus, the behavior of the powers for the incident waves against the incident angle, the frequency and LHM thickness is computed and presented in numerical results with the emphasis on the dissipation factor of the LHM.

\section{THEORY}

We consider four regions each with permittivity $\varepsilon_{\ell}$ and permeability $\mu_{\ell}$, where $\ell$ represents the region order. Region 1 and 4 are vacuums $\left(\varepsilon_{0}, \mu_{0}\right)$, Region 2 is a regular dielectric $\left(\varepsilon_{2}, \mu_{2}\right)$, Region 3 is a metamaterial $\left(\varepsilon_{3}(\omega), \mu_{3}(\omega)\right)$. A polarized plane wave in Region 1 incident on the plane $\mathrm{z}=0$ at some angle $\theta$ relative to the normal to the boundary (see Figure 1).

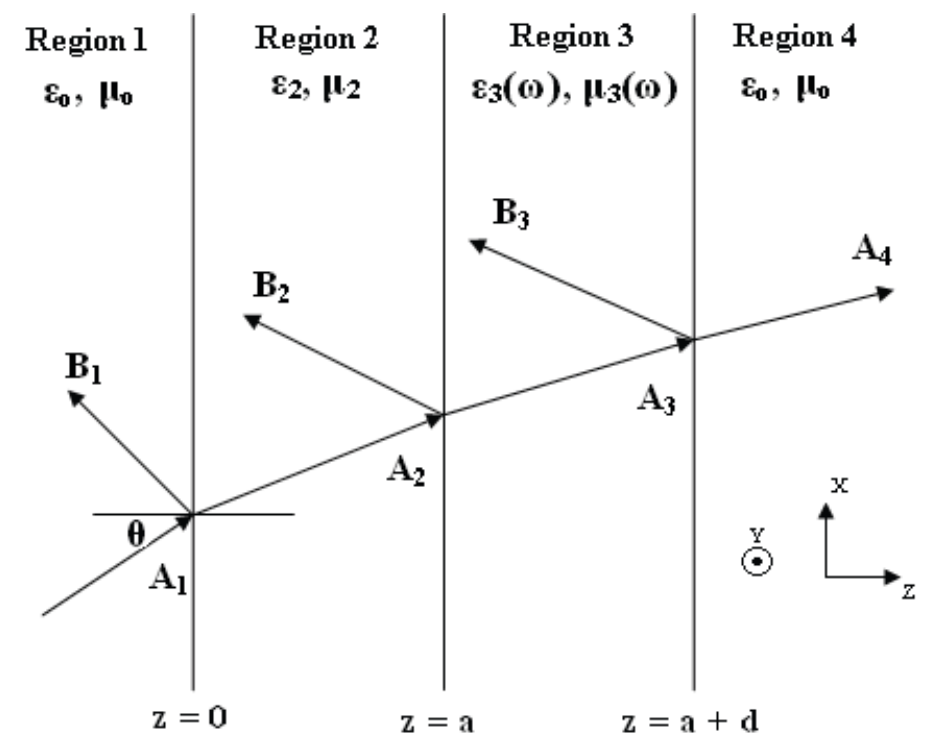

Figure 1. Wave propagation through a structure consisting of a pair of dielectric and metamaterial embedded in vacuum.

The electric field in each region is $[7,11]$ :

$$
\vec{E}_{\ell}=\left(A_{\ell} e^{i k_{\ell z} z}+B_{\ell} e^{-i k_{\ell z} z}\right) e^{i\left(k_{\ell x} x-\omega t\right)} \hat{y}
$$

We use Maxwell's equation as done by [16] to find corresponding magnetic field $\vec{H}_{\ell}$ :

$$
\vec{H}_{\ell}=\frac{1}{\mu_{\ell} \omega}\left[\left(A_{\ell} k_{\ell x} e^{i k_{\ell z} z}+B_{\ell} k_{\ell x} e^{-i k_{\ell z} z}\right) \hat{z}+\left(-A_{\ell} k_{\ell z} e^{i k_{\ell z} z}+B_{\ell} k_{\ell z} e^{-i k_{\ell z} z}\right) \hat{x}\right] e^{i\left(k_{\ell x} x-\omega t\right)}
$$

Where $A_{\ell}$ and $B_{\ell}$ are the amplitudes of forward and backward traveling waves. $k_{\ell}=n_{\ell} \omega / c$ is the wave vector inside the material and $n_{\ell}$ is the refractive index of it. Matching the boundary conditions for $\vec{E}$ and $\vec{H}$ fields at each layer interface, that is at $\mathrm{z}=0, E_{1}=E_{2}$ and $H_{1}=H_{2}$ and so on. This yields six equations with six unknown parameters $[11,13,16]$ : 


$$
\begin{gathered}
A_{1}+B_{1}=A_{2}+B_{2} \\
\frac{k_{1 z}}{\mu_{1}}\left(A_{1}-B_{1}\right)=\frac{k_{2 z}}{\mu_{2}}\left(A_{2}-B_{2}\right) \\
A_{2} e^{i k_{2 z} d_{2}}+B_{2} e^{-i k_{2 z} d_{2}}=A_{3} e^{i k_{3 z} d_{2}}+B_{3} e^{-i k_{3 z} d_{2}} \\
\frac{k_{2 z}}{\mu_{2}}\left(A_{2} e^{i k_{2 z} d_{2}}-B_{2} e^{-i k_{2 z} d_{2}}\right)=\frac{k_{3 z}}{\mu_{3}}\left(A_{3} e^{i k_{3 z} d_{2}}-B_{3} e^{-i k_{3 z} d_{2}}\right) \\
A_{3} e^{i k_{3 z}\left(d_{2}+d_{3}\right)}+B_{3} e^{-i k_{3 z}\left(d_{2}+d_{3}\right)}=A_{4} e^{i k_{4 z}\left(d_{2}+d_{3}\right)} \\
\frac{k_{3 z}}{\mu_{3}}\left(A_{3} e^{i k_{3 z}\left(d_{2}+d_{3}\right)}-B_{3} e^{-i k_{3 z}\left(d_{2}+d_{3}\right)}\right)=\frac{k_{4 z}}{\mu_{4}} A_{4} e^{i k_{4 z}\left(d_{2}+d_{3}\right)}
\end{gathered}
$$

Where $k_{1 x}=k_{2 x}=k_{3 x}=k_{4 x} \equiv$ Snell's law and $k_{\ell z}$ is given by:

$$
k_{\ell z}=\frac{\omega}{c} \sqrt{n_{\ell}{ }^{2}-n_{1}^{2} \sin ^{2} \theta_{1}}
$$

Letting $A_{1}=1$ and solving the obtained equations for the unknown parameters enables us to calculate the reflection and transmission coefficients $B_{1}$ and $A_{4}[11,16]$. The reflected power $\mathrm{R}$ and the transmitted power $\mathrm{T}$ are given by $[11,16]$ :

$$
R=B_{1} B_{1}^{*}, T=\left(k_{4 z} / k_{1 z}\right) A_{4} A_{4}^{*}
$$

Where $B_{1}{ }^{*}$ and $A_{4}{ }^{*}$ are the complex conjugate of $B_{1}$ and $A_{4}$ respectively.

The law of conservation of energy is given by $[11,14,15]$ :

Where, $\mathrm{P}_{\text {loss }}$ is the loss power due to losses in LHM.

$$
R+T=1-\mathrm{P}_{\text {loss }}
$$

\section{THE EFFECTIVE PARAMETERS}

For the LHM in region 3 we employ a dispersive one with $\varepsilon_{3}$ and $\mu_{3}$ appeared in $[2,3,16]$ :

$$
\begin{gathered}
\varepsilon_{3}(\omega)=1-\frac{F_{e} \omega_{e p}^{2}}{\omega^{2}-\omega_{e o}^{2}+i \gamma_{e} \omega} \\
\mu_{3}(\omega)=1-\frac{F_{m} \omega_{m p}^{2}}{\omega^{2}-\omega_{m o}^{2}+i \gamma_{m} \omega}
\end{gathered}
$$

where $\omega_{e p}$ and $\omega_{m p}$ are the electric and magnetic plasma frequencies, $\omega_{e o}$ and $\omega_{m o}$ are the electric and magnetic resonance frequencies. $F_{e}$ and $F_{m}$ are the scaling filling parameters. $\gamma_{\mathrm{e}}$ and $\gamma_{\mathrm{m}}$ are the electric and magnetic dissipation factors. We have used the following parameters appearing in [16]: $\omega_{m p}=2 \pi 10.95 \mathrm{GHz}, \omega_{m o}=2 \pi 10.1 \mathrm{GHz}, F_{m}=.26$, $\omega_{e p}=2 \pi 13.3 \mathrm{GHz}, \omega_{e o}=2 \pi 10.3 \mathrm{GHz}, F_{e}=.37$. The electric and dissipation factors are selected to be equal to each other $\left(\gamma_{e}=\gamma_{m}=\gamma\right)$ and two cases of the LHM are considered, loss-less case $(\gamma=0)$ and loss_case $(\gamma \neq 0)$. The operating frequency is assumed to be $f_{o}=11 \mathrm{GHz}$. This frequency is chosen by an arbitrary decision, but it must be in the frequency band where the permittivity and permeability of the LHM are both simultaneously negative. Region 1, 2 and 4 in Fig. 1 are assumed to be loss-less and the permeability's of them are equal to the permeability of free space. The refractive index of the dielectric in region 4 is selected to be 1.883. The thickness of each of LHM and dielectric slabs is equal to one half-wavelength long at the operating frequency. 


\section{CONSTRUCTION OF THE PROGRAMME}

In this section the MAPLE programme is constructed as follows (note that, only the blue and red items appear in the programme):

- Input

The thickness of the slabs (a, d), the velocity of light $(\mathrm{c}=.3 \mathrm{Gm} / \mathrm{sec})$ as well as the constant $\pi$ :

$>\mathrm{a}:=.0135 ;: \mathrm{d}:=.0135 ;: \mathrm{pi}:=22 . / 7 .: \mathrm{c}:=.3$ :

$$
\begin{aligned}
& \mathrm{a}:=.0135 \\
& \mathrm{~d}:=.0135
\end{aligned}
$$

The frequency and the angle of incidence:

$>$ th: $=30 . *(22 / 7) / 180 .: \mathrm{w}:=2 . * \mathrm{pi}^{*} 11 . ;$ :

$$
\mathrm{W}:=69.14285715
$$

The constants $\gamma_{\mathrm{m}}, \gamma_{\mathrm{e}}, \mathrm{F}_{\mathrm{m}}, \omega_{\mathrm{mp}}$ and $\omega_{\mathrm{mo}}$

$>$ gamam: $=.1$ :gamae $:=.1:$ fm: $=.26:$ wmp: $=2 .{ }^{*}$ pi*10.95:wmo: $=2 .{ }^{*}$ pi*10.1:

The permeability $\mu_{3}$ :

$>$ mu3:=1.-fm* $\mathrm{wmp}^{\wedge} 2 /\left(\mathrm{w}^{\wedge} 2-\mathrm{wmo}^{\wedge} 2+\mathrm{I}^{*} \mathrm{gamam}^{*} \mathrm{w}\right) ;:$

$$
\text { mu3 }:=-.641495669+.01512700063 \mathrm{I}
$$

The permeabilities $\mu_{1,}, \mu_{2}$, and $\mu_{4:}$

$>$ mu1:=1.:mu $4:=1 .: \mathrm{mu} 2:=1$. :

The constants $\mathrm{F}_{\mathrm{e}}, \omega_{\mathrm{ep}}$ and $\omega_{\mathrm{eo}}$ :

$>$ fe:=.37:wep:=2.*pi*13.3:weo:=2.*pi*10.3:

The permittivity $\varepsilon_{3}$ :

$>$ eps $3:=1 .-$ fe*wep $^{\wedge} 2 /\left(\mathrm{w}^{\wedge} 2-\mathrm{weo}^{\wedge} 2+\mathrm{I}^{*} \mathrm{gamae}^{*} \mathrm{w}\right) ;:$

$$
\text { eps } 3:=-3.389019785+.05151431672 \text { I }
$$

The refractive index $\mathrm{n}_{3}$ :

$>\mathrm{n} 31:=\operatorname{sqrt}(\mathrm{mu} 3 * \operatorname{eps} 3) ;$

$$
\mathrm{n} 31:=1.474476067-.02859046592 \text { I }
$$

$>$ n32:=-sqrt(mu3*eps3);:

$$
\mathrm{n} 32:=-1.474476067+.02859046592 \mathrm{I}
$$

To choose the negative sign of $n_{3}$ if $\varepsilon_{3}$ and $\mu_{3}$ are both negative:

$>\mathrm{n} 3:=1 *\left(0+{ }^{\prime} \mathrm{if}^{\prime}(\operatorname{Re}(\mathrm{mu} 3)<0, \mathrm{n} 32, \mathrm{n} 31)\right) ;:$

The refractive indices $\mathrm{n}_{1}, \mathrm{n}_{2}$ and $\mathrm{n}_{4}$ :

$$
\mathrm{n} 3:=-1.474476067+.02859046592 \mathrm{I}
$$

$>\mathrm{n} 1:=1 . \mathrm{n} 2:=1.883: \mathrm{n} 4:=1$. :

The wave vector components in the $\mathrm{z}$ direction:

$>\mathrm{k} 1 \mathrm{z}:=(\mathrm{w} /(\mathrm{c}))^{*} \operatorname{sqrt}\left(\mathrm{n} 1^{\wedge} 2-\mathrm{n} 1^{\wedge} 2 * \sin (\mathrm{th})^{\wedge} 2\right) ;:$

$$
\begin{aligned}
& \mathrm{k} 1 \mathrm{z}:=199.5739453 \\
& >\mathrm{k} 2 \mathrm{z}:=(\mathrm{w} /(\mathrm{c}))^{*} \operatorname{sqrt}\left(\mathrm{n} 2^{\wedge} 2-\mathrm{n} 1^{\wedge} 2^{*} \sin (\mathrm{th})^{\wedge} 2\right) ;: \\
& \mathrm{k} 2 \mathrm{z}:=418.3956406 \\
& >\mathrm{k} 3 \mathrm{z} 1:=(\mathrm{w} /(\mathrm{c}))^{*} \operatorname{sqrt}\left(\mathrm{n} 3^{\wedge} 2-\mathrm{n} 1^{\wedge} 2 * \sin (\mathrm{th})^{\wedge} 2\right) ;: \\
& \mathrm{k} 3 \mathrm{z} 1:=319.6899492-7.004580197 \mathrm{I} \\
& >\mathrm{k} 3 \mathrm{z} 2:=-(\mathrm{w} /(\mathrm{c}))^{*} \operatorname{sqrt}\left(\mathrm{n} 3^{\wedge} 2-\mathrm{n} 1^{\wedge} 2^{*} \sin (\mathrm{th})^{\wedge} 2\right) ;: \\
& \mathrm{k} 3 \mathrm{z} 2:=-319.6899492+7.004580197 \mathrm{I} \\
& >\mathrm{k} 3 \mathrm{z}:=1 *\left(0+{ }^{\mathrm{if}}{ }^{\prime}(\operatorname{Re}(\mathrm{mu} 3)<0, \mathrm{k} 3 \mathrm{z} 2, \mathrm{k} 3 \mathrm{z} 1)\right) ;: \\
& \mathrm{k} 3 \mathrm{z}:=-319.6899492+7.004580197 \mathrm{I} \\
& >\mathrm{k} 4 \mathrm{z}:=(\mathrm{w} /(\mathrm{c}))^{*} \operatorname{sqrt}\left(\mathrm{n} 4^{\wedge} 2-\mathrm{n} 1^{\wedge} 2^{*} \sin (\mathrm{th})^{\wedge} 2\right) ;: \\
& \mathrm{k} 4 \mathrm{z}:=199.5739453
\end{aligned}
$$

The equations 3-8 are inserted:

$>$ solve $(\{\mathrm{A} 1+\mathrm{B} 1=\mathrm{A} 2+\mathrm{B} 2, \mathrm{k} 1 \mathrm{z} / \mathrm{mu} 1 *(\mathrm{~A} 1-\mathrm{B} 1)=\mathrm{k} 2 \mathrm{z} / \mathrm{mu} 2 *(\mathrm{~A} 2-\mathrm{B} 2), \mathrm{A} 2 * \exp (\mathrm{I} * \mathrm{k} 2 \mathrm{z} * \mathrm{a})+\mathrm{B} 2 * \exp (-$ $\left.I * k 2 z^{*} a\right)=A 3 * \exp \left(I * k 3 z^{*} a\right)+B 3 * \exp \left(-I * k 3 z^{*} a\right), k 2 z / m u 2 *\left(A 2 * \exp \left(I * k 2 z^{*} a\right)-B 2 * \exp (-\right.$ $I * k 2 z * a))=k 3 z / m u 3 *(A 3 * \exp (I * k 3 z * a)-B 3 * \exp (-I * k 3 z * a)), A 3 * \exp (I * k 3 z *(a+d))+B 3 * \exp (-$ $\left.\mathrm{I} * \mathrm{k} 3 \mathrm{z}^{*}(\mathrm{a}+\mathrm{d})\right)=\mathrm{A} 4 * \exp (\mathrm{I} * \mathrm{k} 4 \mathrm{z} *(\mathrm{a}+\mathrm{d})), \mathrm{k} 3 \mathrm{z} / \mathrm{mu} 3 *\left(\mathrm{~A} 3 * \exp \left(\mathrm{I} * \mathrm{k} 3 \mathrm{z}^{*}(\mathrm{a}+\mathrm{d})\right)-\mathrm{B} 3 * \exp (-\right.$ $\left.\left.\left.\left.\mathrm{I} * \mathrm{k} 3 \mathrm{z}^{*}(\mathrm{a}+\mathrm{d})\right)\right)=\mathrm{k} 4 \mathrm{z} / \mathrm{mu} 4 * \mathrm{~A} 4 * \exp (\mathrm{I} * \mathrm{k} 4 \mathrm{z} *(\mathrm{a}+\mathrm{d})), \mathrm{A} 1=1\right\},\{\mathrm{A} 1, \mathrm{~B} 1, \mathrm{~A} 2, \mathrm{~B} 2, \mathrm{~A} 3, \mathrm{~B} 3, \mathrm{~A} 4\}\right)$;

- Output 


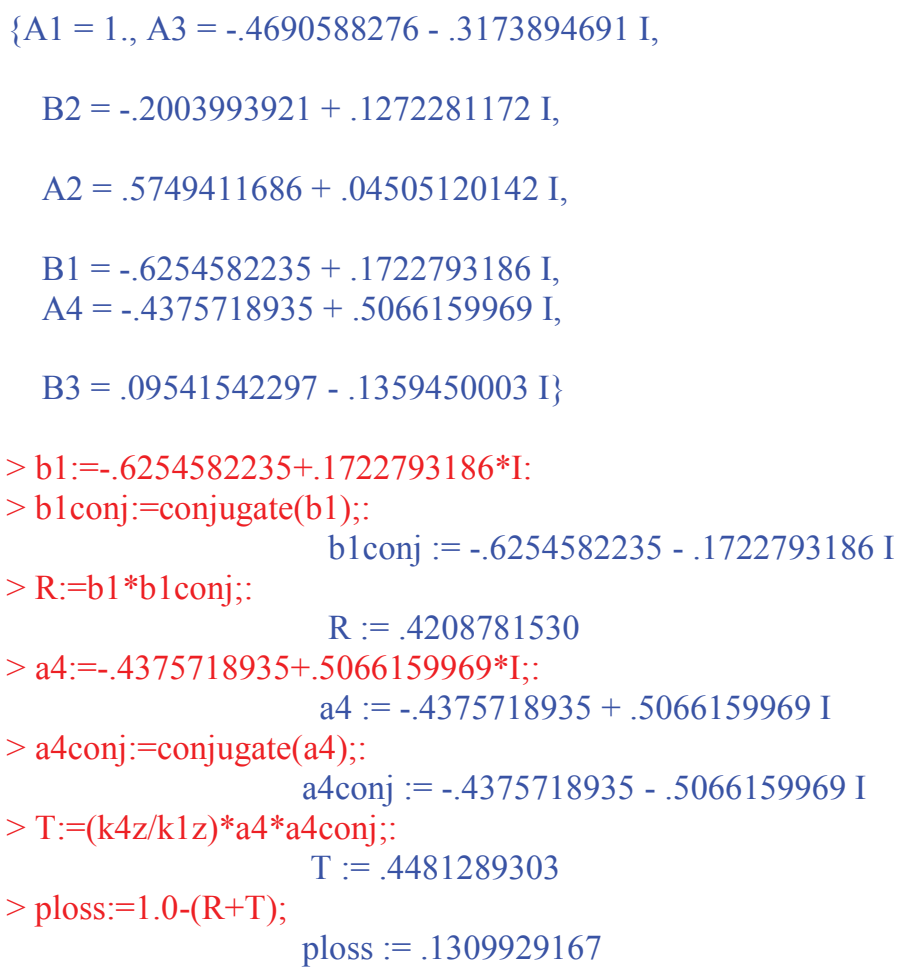

\section{NUMERICAL RESULTS}

In figure 2 we show a calculation of reflected, transmitted and loss powers of the considered structure as a function of frequency for three values of dissipation factor of the LHM $(\gamma=0, .1$ and $.2 \mathrm{GHz})$ and for $30^{\circ}$ angle of incidence. The frequency is changed between $9 \mathrm{GHz}$ and $12 \mathrm{GHz}$, because the simultaneously negative permittivity and permeability can be realized in this range in all cases, according to Eq (12) and (13). As confirmed from Fig. 2, the powers show increasing, decreasing and oscillatory behaviors in different frequency ranges. The interpretation of this is as follows: the transmission is null above $11.5 \mathrm{GHz}$ frequency, where all incident radiation is being reflected. In this band $\varepsilon_{3}(\omega)$ and $\mu_{3}(\omega)$ are different in signs, and then $\mathrm{n}_{3}$ is imaginary, this is because $n_{3}=\sqrt{\varepsilon_{.3} \mu_{3} / \varepsilon_{0} \mu_{0}}$. In 10.3-11.5 GHz band the transmission is very good. In this band $\varepsilon_{3}(\omega)$ and $\mu_{3}(\omega)$ are both negative, and then $\mathrm{n}_{3}$ is real. At 10.1 and 10.2 GHz frequencies the transmitted power is zero because $\mu_{3}(\omega)$ is positive and $\varepsilon_{3}(\omega)$ is negative and then $n_{3}$ is imaginary. In 9-10 GHz band the transmitted power is not zero because both $\varepsilon_{3}(\omega)$ and $\mu_{3}(\omega)$ are positive and then $n_{3}$ is real. This means that electromagnetic waves will only propagate in a medium that has a real index of refraction [16]. This interpretation is presented for loss-less case and can be applied on the loss case of LHM.

Figure 3 illustrates the variation of reflected, transmitted and loss powers with the angle of incidence. The angle of incidence is changed between $0^{\circ}$ and $90^{\circ}$ to realize all possible angles of incidence. Clearly the reflected power increases while the transmitted and loss powers decrease with the angle of incidence. At $90^{\circ}$ the reflected power is maximum while the transmitted and loss powers are minimum at that angle for any value of dissipation factor. The role of the dissipation factor is clear at angles below $90^{\circ}$. The reflected and transmitted powers decrease while the loss power increases with the dissipation factor for any angle below $90^{\circ}$. 


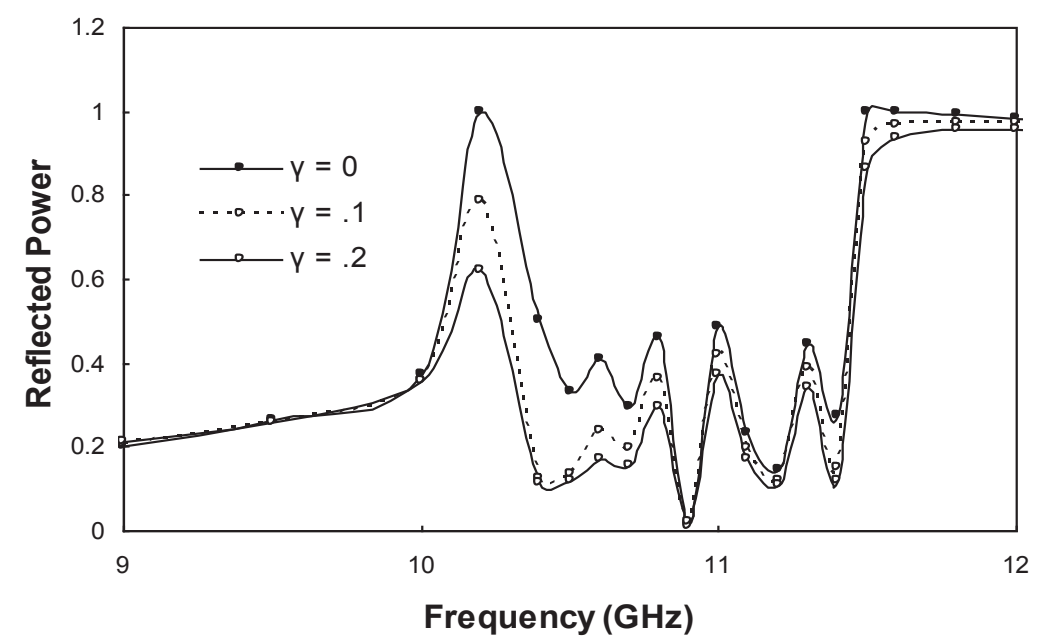

(a)

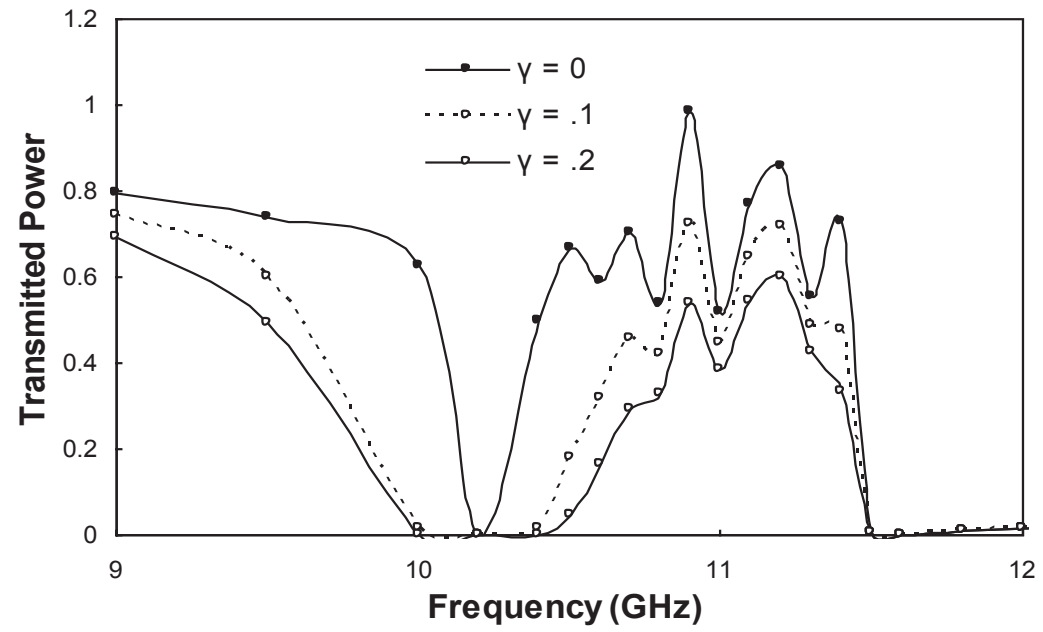

(b)

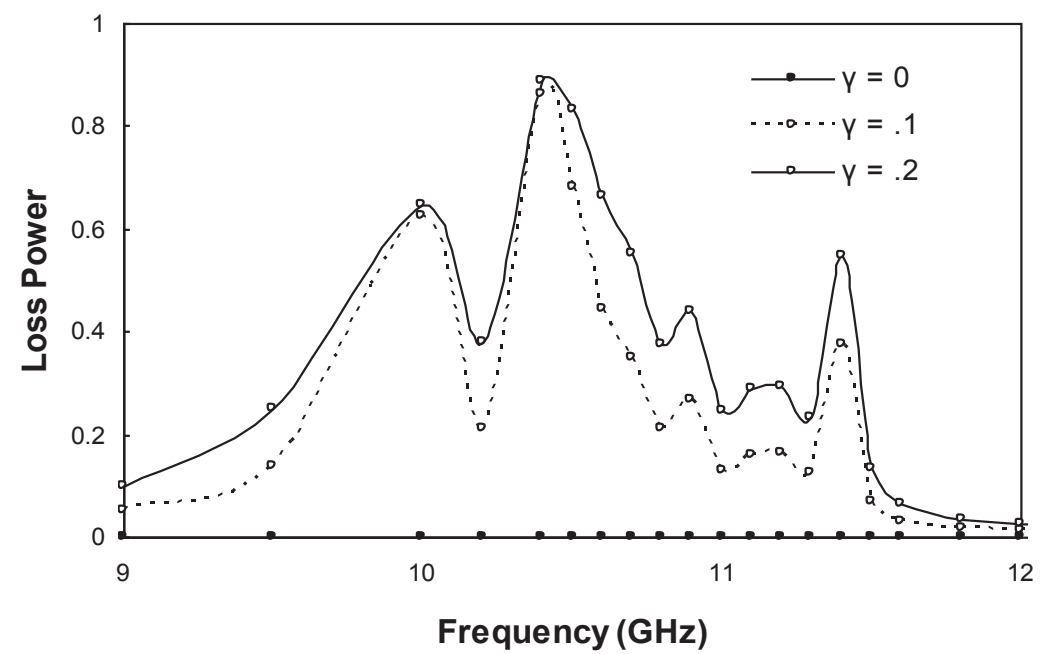

(c)

Figure 2 . Reflected, transmitted and loss powers as a function of frequency.

Proc. of SPIE Vol. 9289 92891L-6 


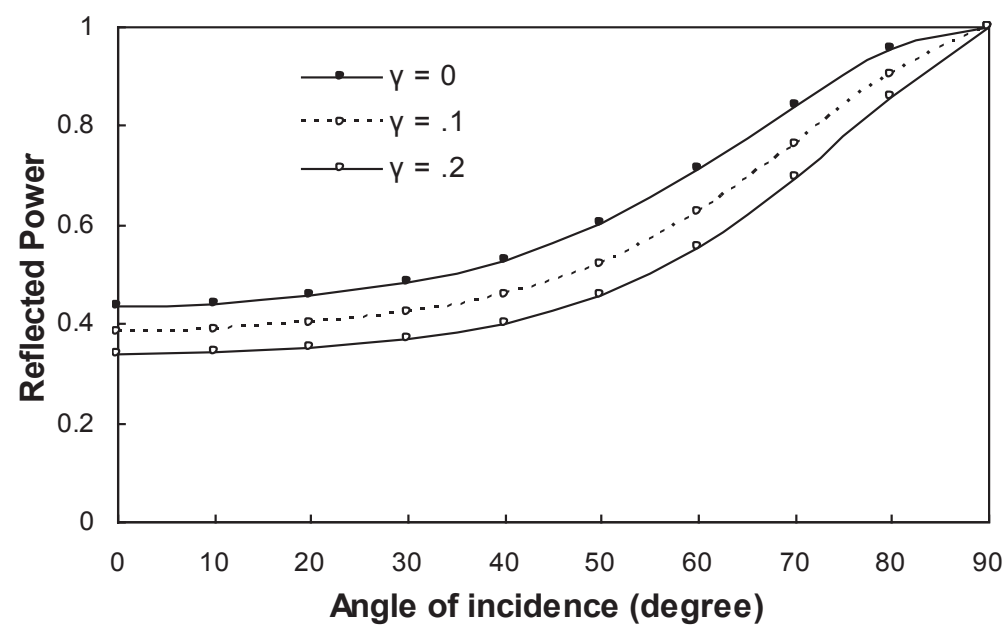

(a)

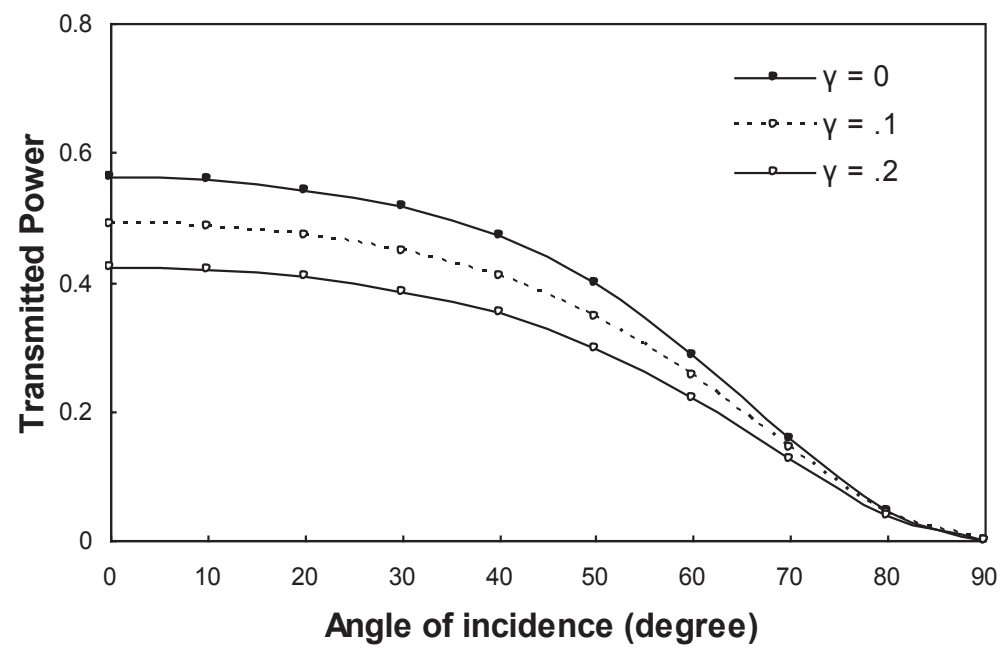

(b)

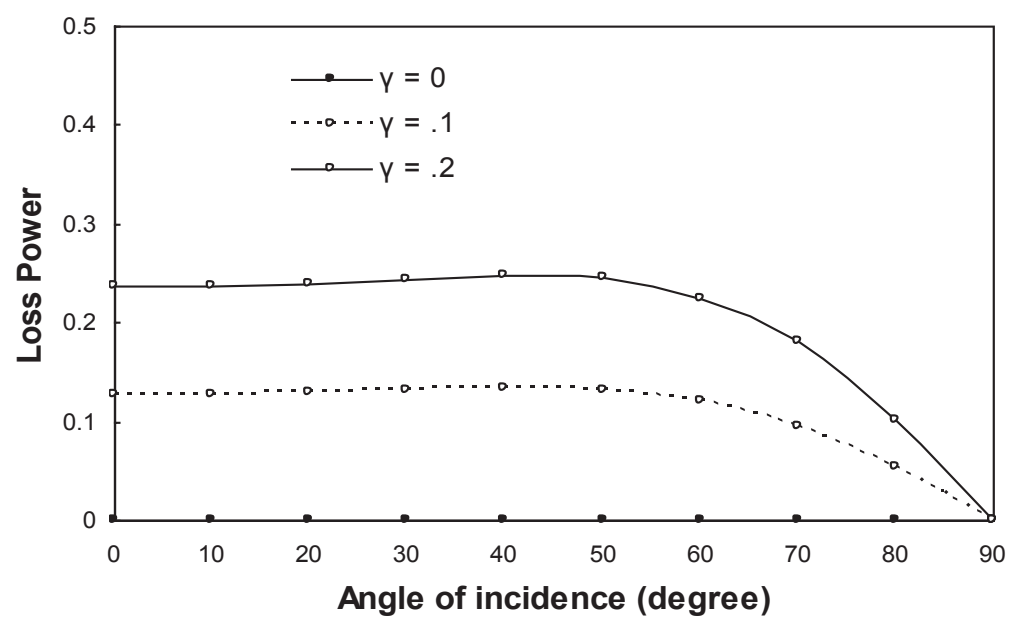

(c)

Figure 3 . Reflected, transmitted and loss powers against the angle of incidence. 


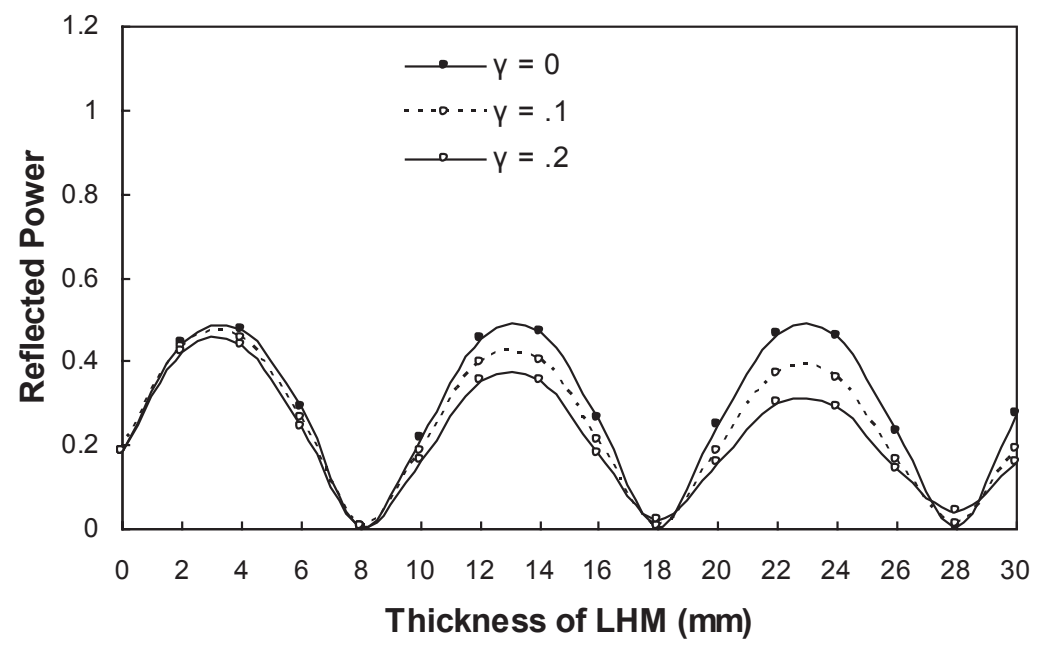

(a)

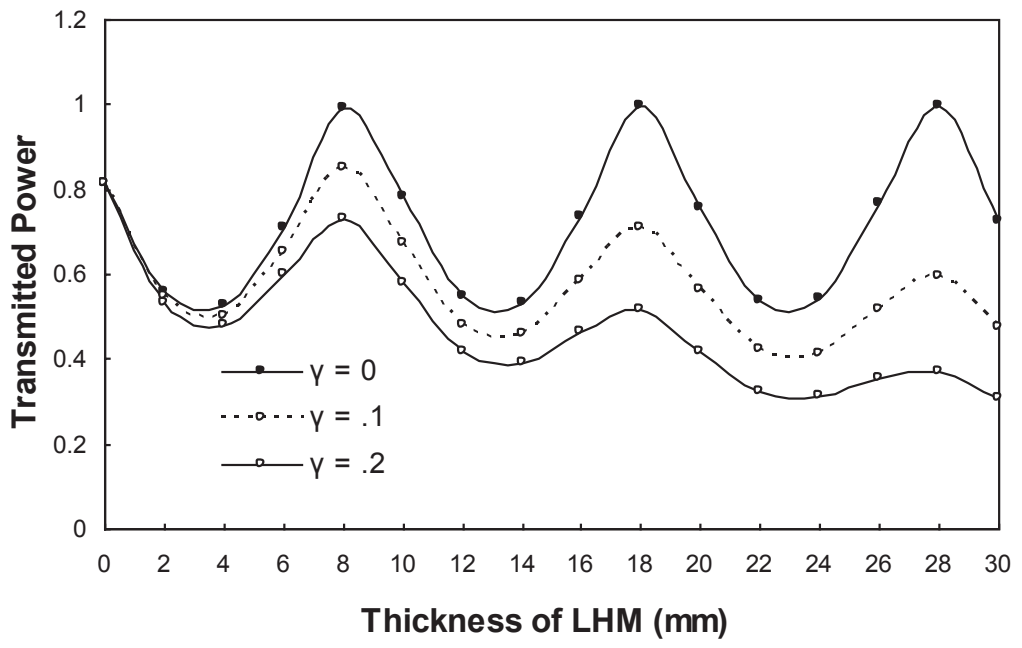

(b)

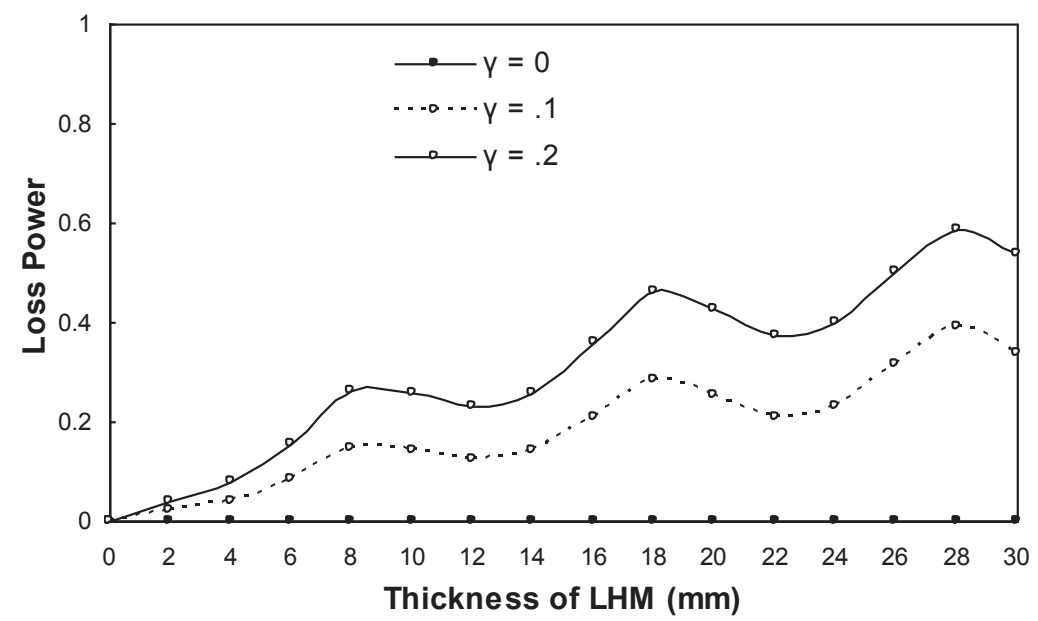

(c)

Figure 4 . Reflected, transmitted and loss powers versus thickness of LHM. 
Figure 4 demonstrates the effect of thickness of LHM on the reflected, transmitted and loss powers respectively at $30^{\circ}$ angle of incidence. The slab thickness is changed from zero to $30 \mathrm{~mm}$ It is noticed from the figure that the reflected power changes periodically for all dissipation factors. The transmitted power shows the same property for $\gamma=0 \mathrm{GHz}$, it shows oscillatory decreasing when $\gamma=.1$ and $.2 \mathrm{GHz}$. On the other hand the loss power shows oscillatory increasing behavior with the increasing values of the dissipation factor.

\section{CONCLUSIONS}

A MAPLE program is designed to study the transmission and reflection of electromagnetic waves by a structure containing LHM with effect of the dissipation factor. The followed method is based on Maxwell's equations and matching the boundary conditions for the electric and magnetic fields at each layer interface. The frequency dependence of $\varepsilon$ and $\mu$ of the LHM is taken into account. The dependence of the reflected, transmitted and loss powers of the considered structure on various parameters have been investigated to observe the effect of the dissipation factor. As can be seen from the numerical results, if the dissipation factor changes, the behaviors of the powers will be affected from this change. Consequently the dissipation factor has an important role in the variations of the powers. The law of conservation of energy given by $[11,14,15]$ is satisfied by our results. The discussed problem is useful for applications which require controlling of reflected and transmitted powers like antenna radome, microwave, millimeter wave and optical devices

\section{REFERENCES}

[1] Veselago, V. G., "The electrodynamics of substances with simultaneously negative values of $\varepsilon$ and $\mu$ ", Soviet Phys Uspekhi 10, 509-514, (1968) .

[2] Pendry, J. B., Holden, A. J., Sewart, W. J. and Youngs, I., "Extremely low frequency plasmons in metallic mesostructure", Phys. Rev. Lett. 76, 4773-4776, (1996).

[3] Pendry, J. B., Holden A. J., Robbins, D. J. and Stewart, W. J., "Magnetic from conductors and enhanced nonlinear phenomena", IEEE Transaction on Microwave Theory and Techniques 47, 2075-2084, (1999).

[4] Shelby, R. A., Smith, D. R. and Schultz, S., "Experimental verification of a negative index of refraction", Science 292, 77-79, (2001).

[5] Shadrivov, I. V., "Nonlinear guided waves and symmetry breaking in left-handed waveguides, Photonic Nanostructure", Fund. Appl. 2, 175-180, (2004).

[6] Garcia, N. and Nieto-Vesperinoas, M., "Left-handed materials do not make a perfect lens", Phys. Rev. Lett. 88, (20)7403, (2002).

[7] Kong, J. A., "Electromagnetic wave interaction with stratified negative isotropic media", Prog. Electromagn. Res. 35, 1-52 (2002).

[8] Mousa, H. M. and Shabat, M. M., "Nonlinear TE surface waves in a left-handed material and magnetic super lattice waveguide structure", Int.J. Mod. Phys. B 21, 895-906, (2007).

[9] Kourakis, I. and Shukla, P. K., "Nonlinear propagation of electromagnetic waves in negative-refraction-index composite materials", Phys. Rev. E Vol.72, 016626 (2005).

[10] Ubeid, M. F., Shabat, M. M. and Sid-Ahmed, M. O., "Effect of negative permittivity and permeability on the transmission of electromagnetic waves through a structure containing left-handed material", Natural Science, 3(4), 328-333, (2011).

[11] Cory, H. and Zach, C., "Wave propagation in metamaterial multi-layered structures", Microwave and Optical Tech. 40, 460-465, (2004).

[12] Sabah, C. and Uckun, S., "High reflection coating with negative and positive refractive index", Piers Online, 5(7), 601-604, (2009).

[13] Oraizi, H. and Abdolali, A., "Mathematical formulation for zero reflection from multilayered metamaterial structures", IEEE Microw. Antennas Progpag., 3, 987-996, (2009).

[14] Stancil, D. D. and Prabhakar, A., Spin waves, Springer, New York (2009).

[15] Sabah, C. and Uckun, S., "Electromagnetic waves propagation through frequency-dispersive and lossy doublenegative slab", Opto-Electron. Rev. 15(3), 133-143, (2007).

[16] Shelby, R. A., Thesis (PhD.), University of Calefornia, San Diego, Microwave Experiments with Left-Handed Materials, Bell and Howell Information and Learning Company (2001). 